\title{
An Overview of Computational Models for Industrial Internet of Things to Enhance Usability
}

\author{
Zhen Ying $\left(\mathbb{D},{ }^{1}\right.$ Iftikhar Ahmad, ${ }^{2}$ Saima Mateen, ${ }^{2}$ Asad Zia, ${ }^{2}$ Ambreen, ${ }^{2}$ Shah Nazir $\mathbb{D},{ }^{2}$ \\ and Neelam Mukhtar ${ }^{3}$ \\ ${ }^{1}$ Party School of Yangzhou Municipal Committee of C.P.C, Yangzhou 225009, China \\ ${ }^{2}$ Department of Computer Science, University of Swabi, Khyber Pakhtunkhwa, Pakistan \\ ${ }^{3}$ College of Home Economics, University of Peshawar, Peshawar, Pakistan
}

Correspondence should be addressed to Zhen Ying; yingzhen1215@sina.com and Shah Nazir; snshahnzr@gmail.com

Received 12 February 2021; Revised 17 June 2021; Accepted 28 July 2021; Published 3 August 2021

Academic Editor: Shahzad Sarfraz

Copyright (c) 2021 Zhen Ying et al. This is an open access article distributed under the Creative Commons Attribution License, which permits unrestricted use, distribution, and reproduction in any medium, provided the original work is properly cited.

In the last decade, the Internet of Things (IoT) has grown to connect a large number of smart entities, devices, and components. These connected entities provide a wide range of services to improve the current society of end customers. The Industrial Internet of Things (IIoTs) are revolutionary systems that have linked manufacturing processes with Internet access in order to preciously increase quality of services. These systems have minimized the costs of production through collaboration with electronic objects, accumulating computing, advanced analytics, and smart perception techniques. A demanding analysis of the strengths and limitations of computational models of IIoT is an essential part of the industry and before deciding which approach to use and implement for enhancing usability. Therefore, the goal of this study is to provide feedback and information to the research community and identify patterns in recommendations for future research in the context of process, development, and monitoring of additional technologies of computational models for IIoT. This paper has presented a comprehensive summary of the existing literature on IIoT for providing details about modern industrial revolutions in the context of IIoT. Associated materials were searched and filtered for identification of relevant materials to the proposed study. These materials have been collectively studied with in-depth analysis and then summarized to condense the information of computation models for the readers as well as entrepreneurs. The study will facilitate research community and practitioners to develop novel techniques, algorithms, and tools to automate and facilitate IIoT. This will develop the field of IIoT and will enhance its usability.

\section{Introduction}

The Industrial Internet was identified as linking equipment, individuals, and statistical analysis on the basis of open widespread network. The description of IIoT aims at improving the intelligence of smart services such as healthcare, manufacturing sites, decreasing energy costs, and enhancing performance through its use. The concept of the IIoT offers a wide variety of IIoT applications in operation and maintenance facilities of life services. Since the current IIoT suggests a similar approach to the system's high-level architecture, the ubiquitous use of the word IoT to contribute its use of emerging technologies in the industry is imprecise because it hinders the study of alternative process models, along with the placement and existence of conventional data processing and related security and performance concerns. The actual world is changed into being digitized and made everything linked to the smart devices. Expansion of smart devices and advancements has permitted humankind to be in steady correspondence at anyplace and anywhere. IoT patterns have made subfragments of the IoT market known as the modern IIoT.

Various approaches have been presented to strengthen the current Industrial IoT concepts and to suggest references for understanding the work of IIoT in various situations, as the commercial internet demanding less delay and high reliability for the transmission rate requires emerging technical assistance such as cloud computing, fog, and fog 
computing. Due to this, the IIoT has attracted worldwide interest, so the creation of the IIoT has become a popular option for potential possibilities and challenges for most industrial services. Sun et al. [1] provide a point of view on how hardware automation can contribute to industry. A portion of the key obstacles are included in the system of successful and adaptable operations. In addition to increasing devices' interoperability, these developments have dispatched the Industry 4.0 operation that is concerned with how digital real frameworks and the web of things can make preparation frameworks flexible, customized, efficient, and secure. In addition, the paper presents how these could be defeated by gear automation to form a real digital computerization network. A good combination of these approaches would consider the vision of Industry 4.0 in the planning of the business. Qin et al. [2] discussed the latest technologies of the Industrial Internet thoroughly, including the design of reference, key developments, relative implementations, and potential difficulties. There are summarized comparison models that have been suggested for different application circumstances and their comparative qualities. A range of applications in the Industrial Internet is supported, and the functional technologies like distributed computing, portable edge processing, and cloud figuring, which are ordered by diverse layers in the model, are implemented. In the meantime, future problems and exploration trends are also addressed to encourage further study of the industrial platform.

The proposed study has presented a comprehensive overview and summarized the recent Industrial Internet of Things literature to provide a detailed analysis of the new industrial revolution in the context of IoT. This research includes an overview of the computational and simulated processes and their applications used in solid state research, providing a shared platform for readers and experimenters.

The rest of the paper is structured in different sections. Section 2 represents the literature review associated with the proposed study. Section 3 outlines a topical guide of computational models in industry in a summarized manner. Section 4 depicts the discussions of the proposed study with details of the existing literature. Section 5 shows the conclusion of the paper, and Section 6 represents the future directions of the study.

\section{Literature Review}

The research community is actively involved in proposing new computational models and approaches for the IIoT. The study establishes an IIoT description and analyses IoT methodologies that are associated. In the current literature, it provides an analysis structure for IIoT, as there is a lack of a comprehensive and analytical review which has focused specifically on this subject of research. Next, it describes the process of applied systematic literature review. The findings and observations are then demonstrated by the study concerning the data obtained. Over the last decade, the Internet of Things has progressed to combine a monstrous number of the issues selecting and consolidating administrations that coordinate the necessary constraints.
Consequently, it has been grouped and analyzed those cutting-edge computations for administration determination under constraints in the IoT environment. Abosaif and Hamza [3] have classified and analyzed the existing algorithms for the selection of service under the constraints of quality of service in the IoT environment. Additionally, they have discussed the potential headings of exploration regarding the strategy, use, and estimation of calculations of fresh aid determination. As of late suggested and being worked on, Boncia et al. [4] explored an architecture with the aim of rendering the design and development of multiagent systems feasible for the vision and the requirement of industry in the systems of cyber-physical era. Most of the standardization research focus is on the IEC 61499 standard considered as a base for the incorporation of industrial agents. Therefore, the study has considered relational multiagent systems as a useful integration aid and a way for research in the area.

To achieve environmental maintainability, the research aims to explore the focal points and obstacles of a scenario configuration approach to sustainable manufacturing industry (SMI) achievement as there is a growing need for the manufacturing industry. Configuration of the situation is a promising approximation to the representation of various thoughts of sustainable man-making projects. The current situation and strategies related to SMIs for this function are analyzed in this paper. A case study analysis of Japan's SMI to 2050, Kishitaa et al. [5], was carried out to examine the potential of a situation plan using an approach of backcasting scenario design. The experimental results of the study depicted that computer-aided scenario design is supportive for the classification of rational and logical structure of the scenarios. In the traditional work process, the basic commissioning aspect of modern control frameworks is carried out on the basis of the actual setup and, thus, during the critical time of the undertaking. Schamp et al. [6] outlined a way to deal with virtual commissioning steps by adding specification and functional details and using formal notations inside the 3D computing system by proper documentation. Design projects within the virtual era represent the conditions, the control logic, and transition in the model. These conditions are constantly encountered during a large charge run of the computing system covering all imaginable state components and so on. The time of reduction in undoubting, dispatching, and prior discovery of value problems will be seen by a limited scope study. Menezes et al. [7] presented a methodology for the identification and design (ID) of smart activities in Industry 4.0 (I4) considering the modern industrial revolution. Industry 4.0 is the fourth industrial revolution which is also known as smart or advanced manufacturing. The elements of the paper were advanced analytics, autonomous robots and system integration, etc. The supposed approach examined four groups of I4 elements. These groups were (i) information and communication technologies, (ii) modelling and solution algorithms, (iii) mechatronics, and (iv) high-performance computing. Small-level industries with large-level systems were studied to find their technological gaps and an example of different firms are also given. At the end, the results were 
tabulated on the bases of these four groups which compare the technologies that are incorporated with the industrial instances.

\section{Computational Approaches in the Industry}

Comprehensive models have been developed for the scientific analysis of industry-related problems and solutions. However, a new exciting age for solving industry-related issues arose with the invention of computers and the advancement of analytical methods, as sophisticated computational techniques were able to provide approximate but substantially precise solutions. Zhu et al. [8] have found that the IoT is highly helpful to get data from a solitary data hub about an object. That being said, in numerous applications, information may be conveyed in various hubs about a solitary object, and the accumulation of data from these hubs has become a typical activity. For this mission, an architecture distributed service-oriented is proposed. Each manufacturer provides support for its own goods in this design, and information hubs preserve the information gathered without anyone else. Semantic inventions are obtained to fix heterogeneity problems and fill in the institution to assist numerous applications. Finally, for example, utilizing this design to address the problem of the next object is illustrated. Figure 1 represents the generic overview of the IIoT. In the figure, various applications, users, servers, application entities, and IoT applications are connected.

3.1. Industry 4.0. Rajput and Singh [9] recommended a mixed-integer linear programming approach for the setup of Industry 4.0 for achieving cleaner production and circular economy through optimizing products-machine allocation. The approach optimizes the trade-off between consumption of energy and processing of machine, cost for gaining cleaner production, and circular economy. The suggested model is further capable of achieving ethical business through sensor deploying for capturing real-time information for establishing the facility of Industry 4.0. Industry 4.0 architecture has been concentrated in countless distributions in the areas of IIoT, enterprise architectures, and also in enterprise integration and cloud manufacturing. An enormous amount of designs had been suggested, yet no one of these had been embraced by countless explorations. Yli-Ojanperä et al. [10] have presented a study with the objectives to overview the studies on Industry 4.0 structures in a manufacturing and survey mindfulness and similarity with Architectural Model Industry 4.0 and architecture of Industrial Internet references. The second objective of the study is to adjust the formerly suggested ideas to Reference Architecture Model Industry 4.0. As for the main objective, it was found that alone a marginal of analysts knew about the said reference models and that by and large practitioners offered no conversation about the similarity of their recommendations with any universally normalized reference engineering for Industry 4.0. Concerning the second objective, it was found that "Reference Architecture Model Industry 4.0" was adult as for correspondence and data partaking in the extent of the associated world, that further normalization empowering the interoperability of various merchants' innovations is as yet a work in progress and that innovation normalization empowering executable business measures between organized undertakings was inadequate.

Rausch et al. [11] presented a container scheduling system enabling the platform for edge setting. The scheduler makes perceptual trade-offs between data and computation development, for example, GPU acceleration, and considers the remaining weighted unambiguous loop requirements. In addition, it has presented a technique to usually fine-tune the weights of scheduling constraints for optimizing high-level objective operations such as limiting of execution task time, availability of uplink, or cost of fog execution. The study has implemented model that targets the Kubernetes action system holder path and submits it to an edge testbed designed. The system is assessed with simulations of trace driven in various infrastructure scenarios through traces produced from processing representative workloads. The experimental results showed the scheduler enhanced the quality of task placement and fine-tuning scheduling parameters supported in meeting operational goals. The advanced IoT applications in the technological world are known as Industry 4.0 and have revitalized the idea of industrial "IoT." The key problems are the authentication and integrity of data as remote correspondence for communication. A few computerized signature plans are suggested in writing to mitigate these difficulties. Nevertheless, such plans experience the adverse effects of inherent key escrow and mystery key appropriation problems due to personalitybased or declared growth. Verma et al. [12] have proposed certificate-based proxy signature approach without pairing. The approach is secure in the random oracle model. The comparison of the performance of computational costs and the length of resulting delegation and signature has shown that the approach cost is appropriate and considerable for the environment of IIoT.

Industry 4.0, named I4.0, marks the fourth in the Industrial Revolution that centers intensely on interconnectivity, independence, AI, and continuous information. By 2020, it is assessed that more than 30 billion of the world's devices will be associated somehow which is a 20 billion with a larger number of devices than today. The steady catching and communicating of information among machines give producing organizations numerous development openings. The IIoT is relied upon changing how we live, work, and play. The main test that looked at the Industrial IoT is security and protection. In the event that we cannot reduce huge amounts of the security and protection given that sway the Industrial IoT, we will not have the option to accomplish its maximum capacity. IoT and the pattern with more noteworthy availability implies that more information accumulated from more places, continuously, to empower ongoing choices and increment income, profitability, and proficiency [13]. 


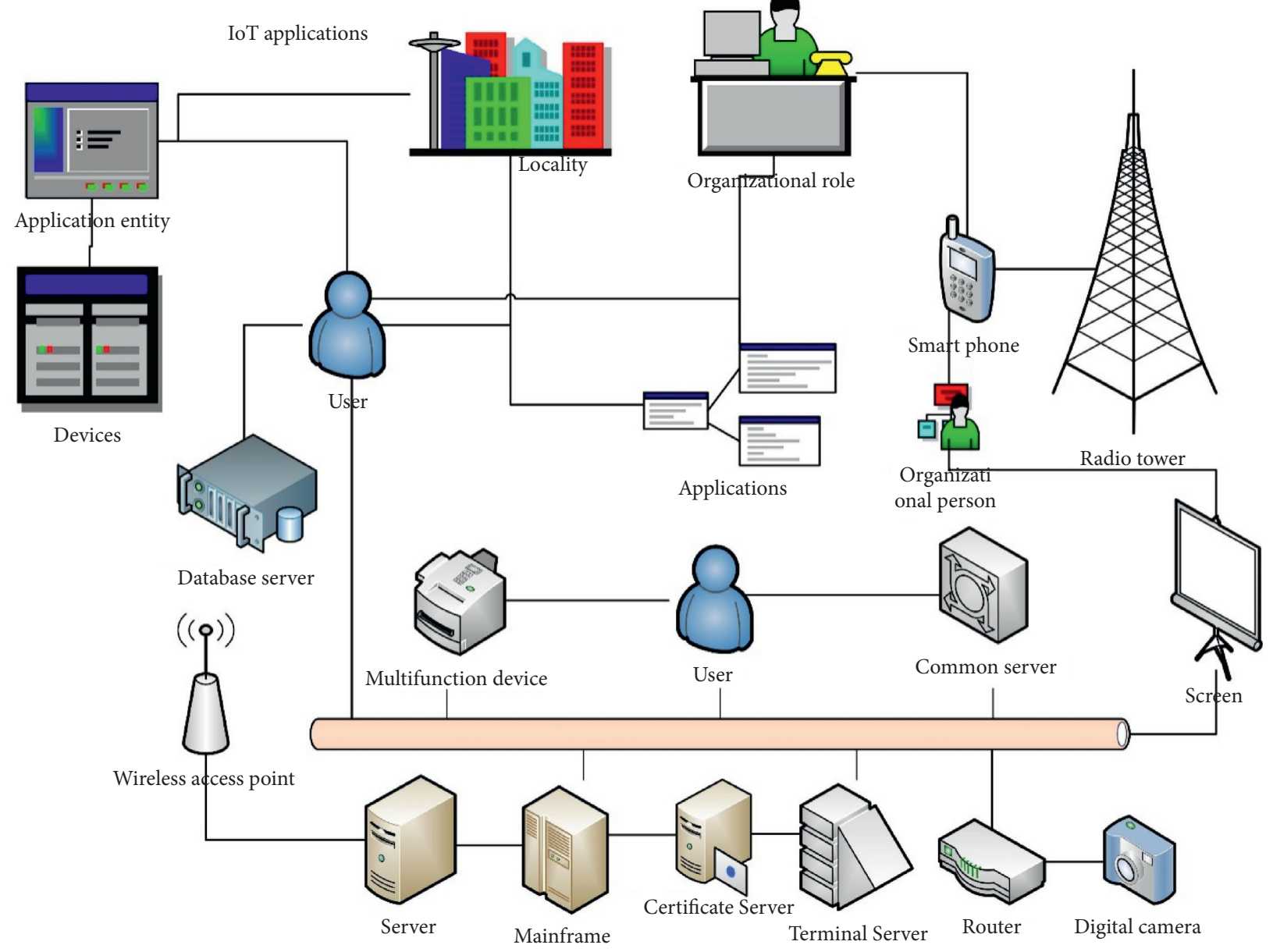

FIgURE 1: Generic overview of IIoT.

\subsection{Vulnerable Automation and Networks Exploitation.} The organizations comprise of industrial materials, services, processes, or manufacturing equipment and wireless devices implanting the activities ongoing for goods during their production period. This suggested control system was formulated by applying a familiar database in the cloud system that controls the process bringing together and manufacturing control logic. This control system has basic uses: configuration of the production system, optimization, control, and monitoring, and historical data storage. The article reports a framework for the implementation and results of the experiments for the assessment of energy. Răileanu et al. [14] have described solution of a semiheterarchical manufacturing control based on infrastructure of cloud for collection of real-time data from smart devices linked to entities of shop-floor. Such entities consist of resources of industry and devices of mobile integrating the work in the process on products for their cycle of manufacturing. Digitalization is generally acknowledged as a primary mechanical system and is relied upon to immensely affect and change industry transcendently by a significant improvement of the whole worth chain. In any case, its execution cycle stays moderate, specifically for little- and medium-sized ventures, as they will in general have misguided judgments about the intricacies and cost of digitalization. Kilimis et al. [15] have presented a study for analyzing factors affecting Brandenburg State, SMEs DEU in the process of their decision making for efficient implementation of digital technologies in their routine business. The response of arising automated patterns and uses of the IoT in recent framework is driven in the direction of the development of IIoT. IIoT covers as an additional vision of IoT in the modern area via computerizing perceptive items for detecting, preparing, imparting, and gathering constant occasions in mechanical frameworks. The significant goal of IIoT is to achieve high operational effectiveness, extended effectiveness, and improved administration of recent resources and cycles by item customization, canny observing applications for creation floor shops and machine wellbeing, and perceptive and precautionary maintenance of mechanical hardware. Khan et al. [16] described the novel and obvious definition of Industrial Internet of Things for supporting the readers in understanding the idea of IIoT. The existing researches in the field are defined in the study. The empowering IIoT technologies and the challenges raised by the IIoT are highlighted. Predictive models are a significant achievement factor for smart manufacturing. In a like manner, simple information-driven models just as mixture models are progressively sent inside the assembling environment for ideal control of plants. However, long-term 
checking and adopting of predictive models has not been a focus point of study up till now; however, it will probably turn out to be progressively more significant as an ever increasing number of predictive models are deployed. Various proposals for successfully overseeing predictive models in smart manufacturing are presented [17].

Digital Twin Technology has the spot in the top 10 vital innovation patterns in 2017 named by Gartner Inc. Advanced Twin idea draws out the virtual or computerized representation of this present reality hardware devices or framework, although this present reality and the virtual world gets the most elevated synchronization. The advanced representation of the total life pattern of an item from its plan stage to the support stage will give the prophetic analysis of the issues in the business. Various models and prediction horizons are analyzed and contrasted to identify the strongest and effective solutions [18]. Brunelli et al. [19] have proposed Deep Learning approach for forecasting production performance in new products packaging. Gao et al. [20] have revisited the collaborative and smart robotic system role and its enabling technologies, incorporating drive system, horizontal integration of robotic network, robotic sensors, friendly and natural interaction of humanrobot, and deep-learnt robots. Intelligence such as computing, motion, cognition, and perception will be effortlessly incorporated for meeting diverse societal and industrial needs. Gittler et al. [21] have presented a novel method for auxiliary units online condition monitoring by considering oil mist separator linked as an example to a 5-axis machine tool. The data were analyzed through the principles of machine learning to remove sufficient condition evaluation, including environmental impacts.

3.3. Cyber-Physical System in Industry. "Internet of Things" related to industries targets to link industrial resources with each other and take advantages from the information that is produced from the resources. In recent years, the broad orchestration of smart devices and the development of technologies related to information and communication are redefining the importance of resources in our mechanism of industry. Social assets are belonging that work together to make the system better optimization. Cyber and physical systems are developed by inserting resources into microcontrollers or computer systems that run actual or real-time results creating algorithms upon the data created from the resources. Bakliwal et al. [22] have proposed an architecture of a multiagent system for collaborative learning and elaborated the results of operation of the architecture for a prognostics issue. IPv6 is the most encouraging convention for conveying network applications in the IoTs and Industry 4.0. While, in any case, its advance selection, specifically in brilliant assembling frameworks, has been much slower than expected. Despite of this reality that the possible main points of IPv6 for modern web of items are noteworthy, it is not efficiently usable. Feldner and Herber [23] presented a qualitative assessment of IPv6 for IIoT. Interviews were led with five experts and users of IPv6. The experimental results indicated that the key issues are tool supporting and available libraries are insufficient, the user has to manually configure the communication of IP, and one-fits-all protocol is prevalent. Some recommendations are presented for further development. Based on trajectory data, the regular behaviour of private cars is extracted [24-26].

Adeodu et al. [27] presented an approach of simulating cavitation in industrial pumps through the technology of IoT. In centrifugal pumps, that is faulty and good, cavitation was simulated through the fast Fourier transform algorithm incorporated to an Arduino microcontroller. Artificial Neural Network was used for numerical analysis of the data. Demartini et al. [28] have built up a theoretical framework which clarifies the potential significance of utilizing computerized innovations on effectiveness, versatility, and manageability. It additionally incorporates evidence from different case analyses, which illustrate the core technologies which would potentiality be able to add to a maintainable industrial future. The discoveries show some impressive outcomes concerning the supportable implications of the digitalization of the assembling process. In the event that the anticipated advantages can be accomplished through computerized advances, they could enormously affect on sustainability.

Guaranteeing an excellent set of model advancement data that precisely represent the manufacturing system is vital to the productive improvement of an information driven model. The cross-business standard cycle process for data mining structure is utilized to give a reference to what exactly stage process manufacturers will confront one unique consideration and difficulties when building up datadriven model. The study at that point investigates how datadriven models can be used to portray, measure streams, and support the implementation of round economy administrators, measure flexibility and waste valorization [29]. Guan et al. [30] have proposed BC-ETS, a safe and proficient BlockChain-based energy trading scheme. In BC-ETS, the energy exchanging model is isolated into two levels, which can secure protection as well as accomplish the harmony between power organic markets. Moreover, to adjust to the generally feeble processing intensity of the hidden IoT hardware in the energy web, a believability-based value evidence instrument is intended to enormously improve the framework accessibility. The examination shows that BCETS can meet the security necessities and has a superior presentation contrasting and other comparable energy exchanging plans. Hansen and Bøgh [31] have presented a comprehensive overview and investigation of how widespread AI and Internet of things are among assembled smalland medium-sized enterprise (SMEs) and talks about the current impediments and openings towards empowering prescient investigation. Right off the bat, a diagram of the empowering influences of $\mathrm{AI}$ and IoT is given along the four examination capacities. From now on, an exhaustive writing audit is led and its discoveries are exhibited. At long last, the arising subjects of innovative work, making AI and IoT open advances to SMEs, and the related future patterns and difficulties are summed up. In the study, end devices are considered here as specialists, which settles on its choice on whether the organization will offload the computation 
undertakings to the edge devices or not. To handle the resource allotment and undertaking offloading, this paper detailed the calculation resource assignment issues as a total cost postponement of this system. An ideal paired computational offloading decision is proposed and afterward fortification learning is acquainted to taking care of the issue. Recreation results show the viability of this support learningbased plan to limit the offloading cost determined as calculation cost and postpone the cost in mechanical web of things situations [32].

The purpose of the study is to evaluate the obstacles which come in the practical accomplishment of Industry 4.0 for the maintained production. The study focuses on eight obstacles which have been shown graphically and are related to each other. The methodology describes the hurdles under supposition of two groups, having names influenced, and aims to lessen them, giving insufficient organizational resources. The two important hurdles according to our study are "technological upgradation" and "lack of policy framework." This study is helpful for little firms and those government investigators who are finding hurdles for maintainable production in Industry 4.0. At the end, they recommend many essential directions for managers and policy makers [33]. The Internet of Things is at the core of the next industrial revolution. That being stated, peer-topeer communication is at the core of industrial IoT, but current industries rely on centralized networking and communication schemes. These centralized networks comply with high standards of IT security. Research has analyzed the industrial communication infrastructure and communication schemes that are already in place and derives the requirements of IT security for the new cloud-based decentralized system. Then, it provides the specification and implementation of the requirements using state of the art communication schemes. Furthermore, the specification and implementations are in strict compliance with IT security standards [34].

3.4. Cyber-Security and Industry. Researches have been done for the identification and mapping of possible vulnerable endpoints in a generic prototype of industry where data will cross through aggregation and suggested an effective way to secure a WSN through ensuring the authenticity and integrity of parcels and identity if the user consists the WSN [35]. Wang et al. [36] have proposed an approach of missing value processing that relies on the combination of generative adversarial and denoising autoencoders aiming at missing completely at a random dataset with high missing rate and noise interference in the scenes of industry. The approach has executed the process of training on a discreate dataset with missing values for ensuring the generated dataset in whole is similar to the distribution of features of the original dataset. Experiments were conducted on various dimensional datasets for proving the feasibility and effectiveness of the approach. Industrial automation and control systems have been increasingly isolated from traditional advanced organizations. Boyes et al. [37] have reviewed the IIoT and their associations with ideas such as cyber-physical systems and Industry 4.0. The study has developed a description of the IIoT and analyzed the associated taxonomies of IoT. An IIoT analysis framework was developed which can be considered for enumerating and characterizing IIoT devices when examining system architectures and security vulnerabilities and threats. $\mathrm{Wu}$ [38] introduced a novel computational framework enabling remote remote real-time sensing, scalable computing, and monitoring for detection and prognosis. The framework uses WSN, machine learning, and cloud computing. A prototype of proof-of-concept was designed for demonstrating how the framework enables manufacturers for monitoring machine health conditions and produce predictive analytics. The experimental results are given for demonstrating the ability and utilization of the framework. Machine learning algorithm was implemented on the public cloud for predicting tool wear in milling operations. The objective of the research is to evaluate IIOT business model in the machine-to-machine (M2M) environment. The study provides a supporting framework concept to classify various types of business models in the M2M environment. Such innovative designs pass by different industries and operate a system firm designs and service applications which are encouraged by smart objects. The supporting concepts can recognize different IIoT business models including (a) company-specific business model, (b) value designs, (c) systemic business model, and (d) systemic value design. It also highlights abstract levels of business models basic unit and dissimilarities among the four business models. At the end, the study advances the concept of "value design" refer to the business model, with different actors coupled together, at last bringing about complex organizations and biological systems of assorted things, cycles, and organizations [39].

Salman et al. [40] presented a study with the aim of showing a way of using augmented reality for retrofitting a production machine. With the help of Microsoft HoloLens, the augmented reality was implemented. The study has briefly described the main technologies of Industry 4.0 and ranking them based on absolute frequency existing in the literature. The research questions defined regarding the association between technologies of Industry 4.0 and productivity. Productivity is considered as the main part of comparison for industry [41]. The research has identified frameworks of open-source machine learning, aligned with the domain of industry for processing data produced from IIoT, in its usage, implementation, programming languages, and future aspects [42]. The current paper presents a first-ofa-kind reference design for creating modern computerization frameworks, dependent anxious edge computing, and blockchain innovations. It additionally presents the design of a platform that implements this reference architecture with the end goal of giving functionality in three reciprocal areas, to be specific, robotization, creation, production systems' virtualization, and data examination. The introduced stage is bound to give some distinct performance and reliability benefits based on its edge computing and blockchain establishment [43]. Kuo et al. [44] have analyzed the relative innovation policy of China, Germany, and USA on the basis of different policy with the aim of industrial 


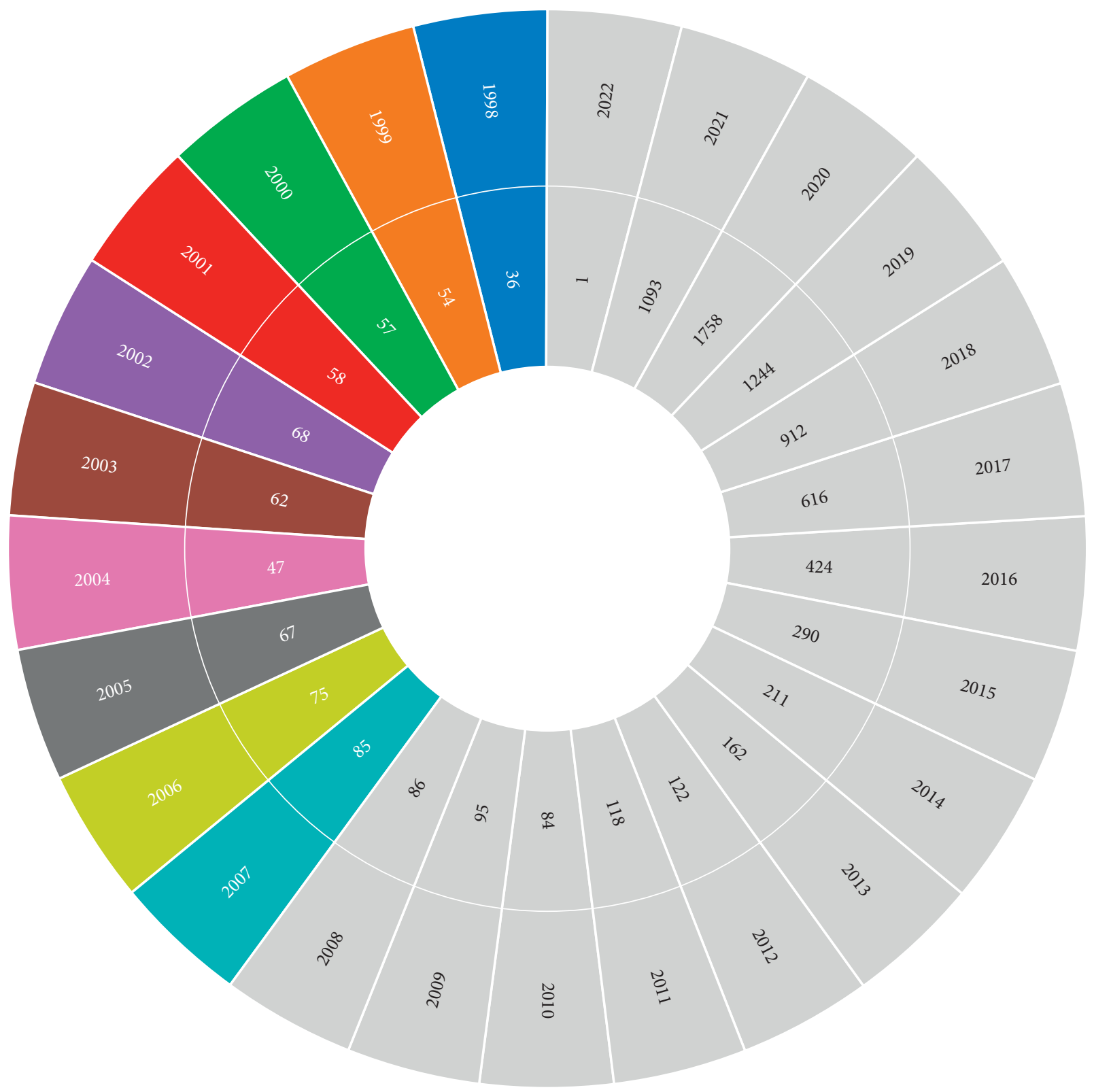

Figure 2: Years of publications and the number of articles published.

revitalization of Industry 4.0. Although the research is a theoretical, a practical approach of Rothwell and Zegveld framework of policy is also adopted. Results have showed that orientation policy of the USA tends to favor demandside policy, political and education, public services, and training policies are favored, whereas policy preferences, public services, technical, scientific development and political favored by Germany and environmental-side policy, political, legal and regulatory, and public service policies by China [44]. Various other approaches have been suggested in the literature in the area of IoT $[45,46]$.

\section{Discussions}

Wide-ranging models and techniques have been proposed for the scientific analysis and management of industry- related problems to overcome their solutions. However, a new exciting age for solving industry-related issues arose with the developments in information technology and the advancement of analytical methods, as sophisticated computational approaches were able to provide imprecise but substantially precise solutions. Research have been done in the area; various approaches, techniques, and solutions were adopted to tackle the issues of modern industry. Figure 2 depicts the years of publications and the number of articles published.

In Figure 2, it is mentioned that with the passage of time, the research in the area is growing and researchers try to come across different solutions from different perspectives. A more number of articles were published in the year 2021, followed by 2020, and so on. This shows the number of researches and its growing rate in the area. The figure depicts 


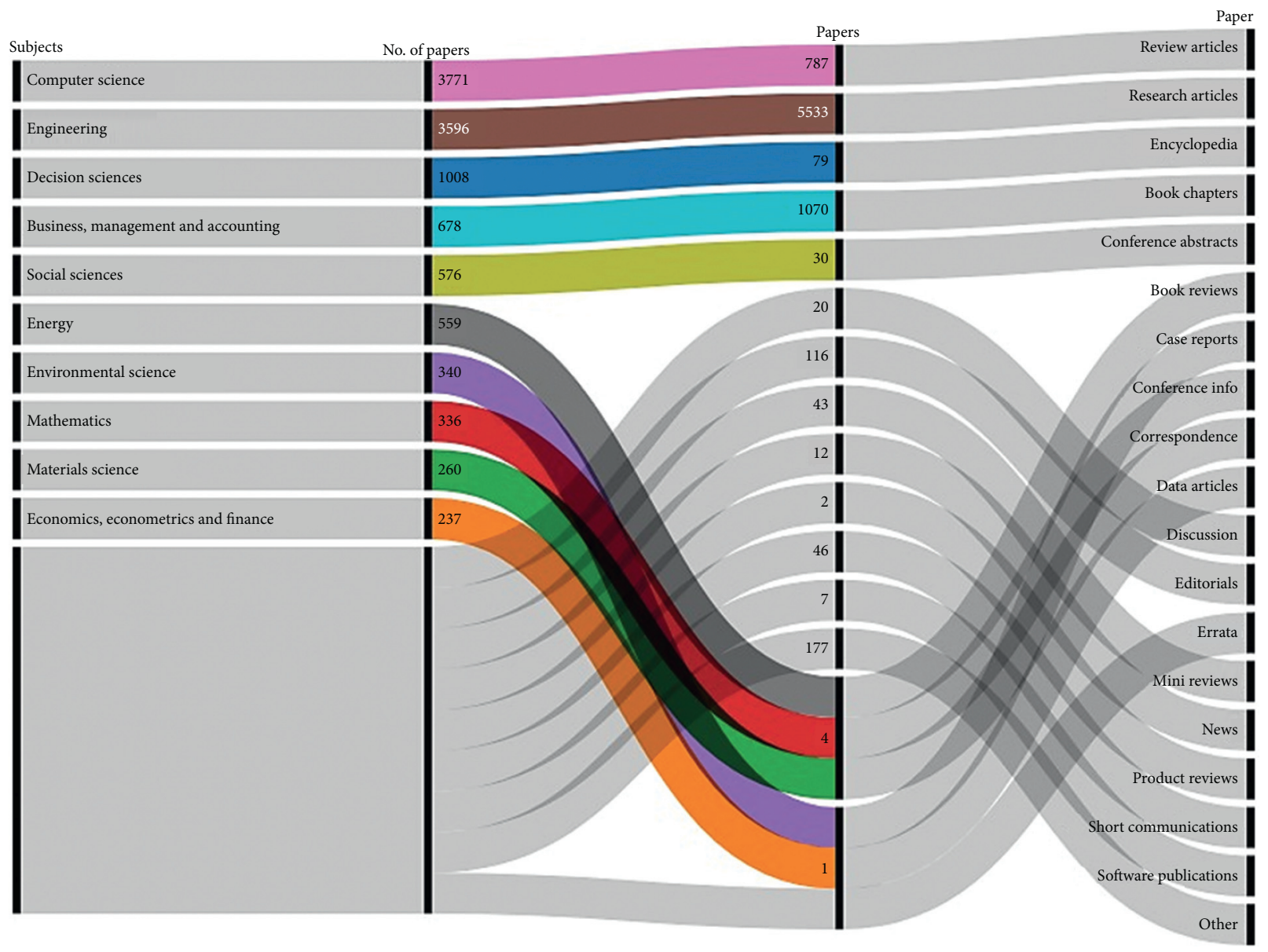

FIgURE 3: Subject, paper types, and the publications numbers.

TABLE 1: Existing approaches related to the proposed work.

\begin{tabular}{|c|c|c|}
\hline Reference & Method & Year \\
\hline$[1]$ & Perspective for equipment automation & 2017 \\
\hline [2] & Recent advances in Industrial Internet & 2019 \\
\hline [3] & Quality of service-aware service selection for IoT & 2020 \\
\hline [4] & RMAS architecture for industrial agents & 2020 \\
\hline [5] & Scenario design to envisioning sustainable manufacturing industries & 2016 \\
\hline$[6]$ & Virtual commissioning of industrial control systems & 2019 \\
\hline [7] & Identification and design of Industry 4.0 & 2019 \\
\hline [10] & Agile manufacturing concept to the reference architecture model Industry 4.0 & 2019 \\
\hline [11] & Optimized container scheduling for data-intensive serverless edge computing & 2021 \\
\hline [12] & Certificate-based proxy signature scheme for IIoT & 2020 \\
\hline [13] & Industry 4.0: IIOT & 2020 \\
\hline [14] & Cloud-based manufacturing control system with data integration from multiple autonomous agents & 2018 \\
\hline [15] & Digitalization for SMEs in Brandenburg, Germany & 2019 \\
\hline [16] & IIoT: recent advances, enabling technologies, and challenges & 2020 \\
\hline [17] & Smart manufacturing and continuous improvement and adaptation of predictive models & 2020 \\
\hline [18] & The industry use cases for the Digital Twin idea & 2020 \\
\hline [19] & Deep learning-based production forecasting in manufacturing & 2020 \\
\hline$[20]$ & From Industry 4.0 to Robotics 4.0 & 2020 \\
\hline [21] & Condition monitoring system for machine tool auxiliaries & 2020 \\
\hline$[22]$ & Multiagent system architecture to implement collaborative learning & 2018 \\
\hline [23] & Qualitative evaluation of IPv6 for IoT & 2018 \\
\hline [27] & IIoTS-based technology for prediction and control of cavitation in centrifugal pumps & 2020 \\
\hline
\end{tabular}


TABle 1: Continued.

\begin{tabular}{|c|c|c|}
\hline Reference & Method & Year \\
\hline [28] & Digitalization technologies for industrial sustainability & 2019 \\
\hline$[29]$ & Considerations, challenges, and opportunities when developing data-driven models for process manufacturing systems & 2020 \\
\hline$[30]$ & Secure and efficient energy trading in IIoT-enabled energy Internet & 2020 \\
\hline$[31]$ & $\mathrm{AI}$ and IoT in small- and medium-sized enterprises & 2020 \\
\hline [33] & Modeling the Industry 4.0 adoption for sustainable production in micro, small, and medium enterprises & 2021 \\
\hline$[34]$ & Securing future decentralized industrial IoT infrastructures & 2019 \\
\hline$[35]$ & Mapping vulnerabilities in IIoT landscape & 2019 \\
\hline [37] & IIoT: an analysis framework & 2018 \\
\hline$[38]$ & Fog computing-based framework for process monitoring and prognosis in cyber-manufacturing & 2017 \\
\hline [39] & IIoT business models in the machine-to-machine context & 2020 \\
\hline$[41]$ & Relationship between Industry 4.0 and productivity & 2019 \\
\hline$[42]$ & Open-source machine learning for IIoT & 2020 \\
\hline$[44]$ & Industrial revitalization via Industry 4.0 & 2019 \\
\hline$[46]$ & Parallel computing for efficient and intelligent IIoHT & 2021 \\
\hline
\end{tabular}

that year-wise the publications are rising. Figure 3 briefly represents the subjects, paper type, and the materials published. The figure contains mainly two different categories including the paper type and its subjects of publications. The figure depicts that more number of articles were published as "research article" followed by "book chapter", and so on. Also, more number of articles were published in the discipline of "computer science."

The existing approaches used in the area of research are given in Table 1. This table represents mainly the reference of the articles published, along with the method and year of publication.

\section{Conclusion}

The IIoT aims at enlightening the intelligence of smart services such as healthcare, smart cities, and other manufacturing sites, decreasing energy costs, and enhancing performance through its use. The idea of the IIoT offers a wide variety of IIoT smart applications in operation and maintenance facilities. The IIoTs are revolutionary systems that have connected manufacturing processes with Internet access in order to affectedly increase quality and minimizes costs of production through collaboration with electronic objects accumulates computing, advanced analytics, and smart perception techniques. A demanding analysis of the strengths and weaknesses of computational models of IIoT is an essential part of the industry and before deciding which ones to implement for enhancing usability of industry is a challenging issue. The goal of this analysis is to facilitate response and information to the research community and recognize patterns of propositions for future research in context of the process, development and monitoring of other technologies. This paper has summarized the existing related work on IIoT for providing a comprehensive review about modern industrial revolutions in the context of IIoT with the support of computational models. Related materials were searched and filtered for identifications of the most important materials to the proposed study. These materials have been studied with in-depth investigation and then summarized to condense the information of computation models for the readers as well as entrepreneurs.

\section{Future Direction}

The contribution of the study is to present a compressive summary of the existing literature on IIoT to provide details about modern industrial revolutions in the context of IIoT. Related researches and study materials were searched and filtered for identification of associated materials. These materials have been collectively studied and analyzed indepth, and then summary of the approaches has been presented to condense the information of computation models. This study will facilitate the research community and practitioners to develop and devise novel techniques, algorithms, solutions, and tools to automate and facilitate modern industrial Internet of Things. This will enhance the developments in the field of IIoT and will improve its usability and effectiveness.

\section{Data Availability}

The data used to support the findings of this study are included within the article.

\section{Conflicts of Interest}

The authors declare that they have no conflicts of interest regarding the publication of this paper.

\section{Acknowledgments}

This study was supported by study on the present situation and control of water ecology in Yangzhou Section of Grand Canal (16XZB023).

\section{References}

[1] B. Sun, S.-L. Jämsä-Jounela, Y. Todorov, L. E. Olivier, and I. K. Craig, "Perspective for equipment automation in process industries," IFAC-PapersOnLine, vol. 502, pp. 65-70, 70.

[2] W. Qin, S. Chen, and M. Peng, "Recent advances in industrial internet: insights and challenges," Digital Communications and Networks, vol. 61, pp. 1-13, 8648.

[3] A. N. Abosaif and H. S. Hamza, "Quality of service-aware service selection algorithms for the internet of things environment: a review paper," Array, Journal, vol. 8, p. 2590, 2020. 
[4] A. Boncia, S. Longhia, E. Lorenzonia, and M. Pirania, "RMAS architecture for industrial agents in IEC 61499," Procedia Manufacturing, vol. 90, no. 42, http://creativecommons.org/ licenses/by-nc-nd/4.0/, 2020.

[5] Y. Kishita, Y. Mizuno, and Y. Umeda, "Scenario design approach to envisioning sustainable manufacturing industries to 2050," Procedia CIRP, vol. 48, pp. 407-412, 2016, http:// creativecommons.org/licenses/by-nc-nd/4.0.

[6] M. Schamp, L. V. D. Ginste, S. Hoedt, A. Claeys, E.-H. Aghezzaf, and J. Cottyn, "Virtual commissioning of industrial control systems - a 3D digital model approach," Procedia Manufacturing, vol. 39, pp. 66-73, 2019.

[7] B. C. Menezes, J. D. Kelly, and A. G. Leal, "Identification and design of industry 4.0 opportunities in manufacturing: examples from mature industries to laboratory level systems," IFAC-PapersOnLine, vol. 52, no. 13, pp. 2494-2500, 2019.

[8] T. Zhu, S. Dhelim, Z. Zhou, S. Yang, and H. Ning, "An architecture for aggregating information from distributed data nodes for industrial internet of things," Computers \& Electrical Engineering, vol. 58pp. 337-349, 349.

[9] S. Rajput and S. P. Singh, "Industry 4.0 Model for circular economy and cleaner production," Journal of Cleaner Production, vol. 277, Article ID 123853, 2020.

[10] M. Yli-Ojanperä, S. Sierla, N. Papakonstantinou, and V. Vyatkin, "Adapting an agile manufacturing concept to the reference architecture model industry 4.0: a survey and case study," Journal of Industrial Information Integration, vol. 15, pp. 147-160, 2019.

[11] T. Rausch, A. Rashed, and S. Dustdar, "Optimized container scheduling for data-intensive serverless edge computing," Future Generation Computer Systems, vol. 114, pp. 259-271, 2021.

[12] G. K. Verma, B. B. Singh, N. Kumar, M. S. Obaidat, D. He, and H. Singh, "An efficient and provable certificate-based proxy signature scheme for IIoT environment," Information Sciences, vol. 518, pp. 142-156, 2020.

[13] S. Munirathinam, "Industry 4.0: industrial internet of things (IIOT)," in Advances in Computers, P. Raj and P. Evangeline, Eds., vol. 117, , pp. 129-164, Elsevier, 2020.

[14] S. Răileanu, F. Anton, T. Borangiu, S. Anton, and M. Nicolae, "A cloud-based manufacturing control system with data integration from multiple autonomous agents," Computers in Industry, vol. 102, pp. 50-61, 2018.

[15] P. Kilimis, W. Zou, M. Lehmann, and U. Berger, "A survey on digitalization for SMEs in Brandenburg, Germany," IFACPapersOnLine, vol. 52, no. 13, pp. 2140-2145, 2019.

[16] W. Z. Khan, M. H. Rehman, H. M. Zangoti, M. K. Afzal, N. Armi, and K. Salah, "Industrial internet of things: recent advances, enabling technologies and open challenges," Computers \& Electrical Engineering, vol. 81, p. 106522, Article ID 106522, 2020.

[17] G. Kronberger, F. Bachinger, and M. Affenzeller, "Smart manufacturing and continuous improvement and adaptation of predictive models," Procedia Manufacturing, vol. 42, pp. 528-531, 2020.

[18] P. Augustine, "The industry use cases for the digital twin idea," in Advances in Computers, P. Raj and P. Evangeline, Eds., vol. 117, pp. 79-105, Elsevier, Amsterdam, Netherlands, 2020.

[19] L. Brunelli, C. Masiero, D. Tosato, A. Beghi, and G. A. Susto, "Deep learning-based production forecasting in manufacturing: a packaging equipment case study," Procedia Manufacturing, vol. 38, pp. 248-255, 2019.

[20] Z. Gao, T. Wanyama, I. Singh, A. Gadhrri, and R. Schmidt, "From industry 4.0 to robotics 4.0 - a conceptual framework for collaborative and intelligent robotic systems," Procedia Manufacturing, vol. 46, pp. 591-599, 2020.

[21] T. Gittler, F. Stoop, D. Kryscio, L. Weiss, and K. Wegener, "Condition monitoring system for machine tool auxiliaries," Procedia CIRP, vol. 88, pp. 358-363, 2020.

[22] K. Bakliwal, M. H. Dhada, A. S. Palau, A. K. Parlikad, and B. K. Lad, "A multi agent system architecture to implement collaborative learning for social industrial assets," IFACPapersOnLine, vol. 51, no. 11, pp. 1237-1242, 2018.

[23] B. Feldner and P. Herber, "A qualitative evaluation of IPv6 for the industrial internet of things," Procedia Computer Science, vol. 134, pp. 377-384, 2018.

[24] Z. Xiao, S. Xu, T. Li et al., "On extracting regular travel behavior of private cars based on trajectory data analysis," IEEE Transactions on Vehicular Technology, vol. 69, no. 12, pp. 14537-14549, 2020.

[25] Y. Huang, Z. Xiao, D. Wang, H. Jiang, and D. Wu, "Exploring individual travel patterns across private car trajectory data," IEEE Transactions on Intelligent Transportation Systems, vol. 21, no. 12, pp. 5036-5050, 2020.

[26] Y. Huang, Z. Xiao, X. Yu, D. Wang, V. Havyarimana, and J. Bai, "Road network construction with complex intersections based on sparsely-sampled private car trajectory data," ACM Transactions on Knowledge Discovery from Data (TKDD), vol. 13, no. 35, pp. 1-28, 2019.

[27] A. Adeodu, I. Daniyan, O. Omitola, C. Ejimuda, E. Agbor, and O. Akinola, "An adaptive Industrial Internet of things (IIOts) based technology for prediction and control of cavitation in centrifugal pumps," Procedia CIRP, vol. 91, pp. 927-934, 2020.

[28] M. Demartini, S. Evans, and F. Tonelli, "Digitalization technologies for industrial sustainability," Procedia Manufacturing, vol. 33, pp. 264-271, 2019.

[29] O. J. Fisher, N. J. Watson, J. E. Escrig et al., "Considerations, challenges and opportunities when developing data-driven models for process manufacturing systems," Computers \& Chemical Engineering, vol. 140, Article ID 106881, 2020.

[30] Z. Guan, X. Lu, N. Wang, J. Wu, X. Du, and M. Guizani, "Towards secure and efficient energy trading in IIoT-enabled energy internet: a blockchain approach," Future Generation Computer Systems, vol. 110, pp. 686-695, 2020.

[31] E. B. Hansen and S. Bøgh, "Artificial intelligence and internet of things in small and medium-sized enterprises: a survey," Journal of Manufacturing Systems, vol. 58, no. 13, pp. 362-372, 2021.

[32] M. S. Hossain, C. I. Nwakanma, J. M. Lee, and D.-S. Kim, "Edge computational task offloading scheme using reinforcement learning for IIoT scenario," ICT Express, vol. 6, no. 4, pp. 291-299, 2020.

[33] A. G. Khanzode, P. R. S. Sarma, S. K. Mangla, and H. Yuan, "Modeling the industry 4.0 adoption for sustainable production in micro, small \& medium enterprises," Journal of Cleaner Production, vol. 279, Article ID 123489, 2021.

[34] S. Plaga, N. Wiedermann, S. D. Anton, S. Tatschner, H. Schotten, and T. Newe, "Securing future decentralised industrial IoT infrastructures: challenges and free open source solutions," Future Generation Computer Systems, vol. 93, pp. 596-608, 2019.

[35] D. Mourtzis, K. Angelopoulos, and V. Zogopoulos, "Mapping vulnerabilities in the industrial internet of things landscape," Procedia CIRP, vol. 84, pp. 265-270, 2019.

[36] H. Wang, Z. Yuan, Y. Chen, B. Shen, and A. Wu, "An industrial missing values processing method based on generating model," Computer Networks, vol. 158, pp. 61-68, 2019. 
[37] H. Boyes, B. Hallaq, J. Cunningham, and T. Watson, "The industrial internet of things (IIoT): an analysis framework," Computers in Industry, vol. 101, pp. 1-12, 2018.

[38] D. Wu, S. Liu, L. Zhang et al., "A fog computing-based framework for process monitoring and prognosis in cybermanufacturing," Journal of Manufacturing Systems, vol. 43, pp. 25-34, 2017.

[39] S. Leminen, M. Rajahonka, R. Wendelin, and M. Westerlund, "Industrial internet of things business models in the machineto-machine context," Industrial Marketing Management, vol. 84, pp. 298-311, 2020.

[40] S. Salman, H. Al-Maeenia, C. Kuhnhena, B. Engela, and M. Schillera, "Smart retrofitting of machine tools in the context of industry 4.0," Procedia CIRP, vol. 88, pp. 369-374, 2020.

[41] S. K. Hubert Backhaus and D. Nadarajah, "Investigating the relationship between industry 4.0 and productivity: a conceptual framework for malaysian manufacturing firms," Procedia Computer Science, vol. 161, pp. 696-706, 2019.

[42] A. I. Khan and A. Al-Badi, "Open source machine learning frameworks for industrial internet of things," Procedia Computer Science, vol. 170, pp. 571-577, 2020.

[43] M. Isaja and J. K. Soldatos, "Distributed ledger architecture for automation, analytics and simulation in industrial environments," IFAC-PapersOnLine, vol. 51, no. 11, pp. 370-375, 2018.

[44] C.-C. Kuo, J. Z. Shyu, and K. Ding, "Industrial revitalization via industry 4.0 - a comparative policy analysis among China, Germany and the USA," Global Transitions, vol. 1, pp. 3-14, 2019.

[45] Q. Hao, S. Nazir, X. Gao, L. Ma, and M. Ilyas, "A review on multicriteria decision support system and industrial internet of things for source code transformation," Scientific Programming, vol. 2021, pp. 1-9, Article ID 6661272, 2021.

[46] X. Yang, S. Nazir, H. U. Khan, M. Shafiq, and N. Mukhtar, "Parallel computing for efficient and intelligent industrial internet of health things: an overview," Complexity, vol. 2021, pp. 1-11, Article ID 6636898, 2021. 